\title{
Utilização de Simulações pelo Método Monte Carlo para Avaliação dos Índices de Confiabilidade num Sistema Fictício de Geração de Energia
}

\author{
Márcio J. Dias, Murillo V. Santos, Hélio S. Queiroz, Jorge M. A. Costa, Rosemberg \\ F. N. Rodrigues, Lorena F. de Souza, Sonimar R. M. Dias \& Vítor S. Menezes
}

As energias renováveis são geradas por meio de recursos naturais, ou seja, para que haja sua produção deve-se causar o menor impacto ambiental possível, sendo as mais conhecidas: hidráulica, biomassa, solar, eólica, geotérmica e maremotriz. No Brasil, devido à enorme quantidade de rios, a maior parte da energia elétrica disponível é produzida pelas grandes usinas hidrelétricas, mas atualmente, a fim de apresentar solução para demanda energética do país, vem crescendo bastante a produção de energia elétrica por meio da biomassa, da irradiância solar e pela força dos ventos. No entanto, as injeções dessas energias produzidas pelas "micros" usinas geradoras podem ocasionar incertezas probabilísticas ao sistema de distribuição e transmissão. O objetivo deste trabalho é avaliar a confiabilidade de um sistema fictício de geração de energia, por meio da implementação da simulação do Método Monte Carlo.

Palavras Chave: $L O L E$; LOLP; EENS.

As renewable energies are generated through natural resources, that is, because their production is more dangerous, being better known: hydraulics, biomass, solar, wind, geothermal and tidal energy. In Brazil, the large number of rivers, solar energy is large and hydroelectric, but nowadays, solar energy, solar energy, solar irradiation and wind energy. However, the injections of the energies produced by the micros can generate the probabilities of the distribution and transmission system. The objective of this work is to guarantee the power generation system, through the implementation of the Monte Carlo method simulation.

Keywords: $L O L E$; LOLP; EENS. 


\section{Introdução}

Energia elétrica é uma forma de energia baseada na geração de diferenças de potencial elétrico entre dois pontos, que permitem estabelecer uma corrente elétrica entre ambos. ${ }^{2}$ É uma das formas de energia mais utilizadas atualmente devido à facilidade do transporte, baixos índices de perdas, durante os processos de conversões. A energia elétrica é obtida por fontes não renováveis (carvão, óleo combustível, gás e outros), por fontes renováveis (hidráulica, biomassa, solar, eólica, geotérmica, maremotriz e hidrogênio), e ainda por combustíveis nucleares radioativos, como por exemplo, - urânio. $^{11}$

No Brasil, devido à enorme quantidade de rios, a maior parte da energia elétrica disponível é produzida pelas grandes usinas hidrelétricas. Essa forma de se produzir energia é considerada como fontes renováveis, pois as águas utilizadas para girar as turbinas são desenvolvidas ao meio ambiente. No entanto, existe grande impacto ambiental ocasionado pelo represamento dessas águas ${ }^{1}$

Além da intenção de diminuir impacto ambiental, causado pelo represamento das águas, atualmente, temse investido em outras formas de geração como: solar, eólica e biomassa, pois a produção de energia por meio de hidrelétricas não está sendo suficiente para atender à demanda do mercado brasileiro. ${ }^{12}$

A produção de energia solar e eólica vem sendo desenvolvida em grande escala nas regiões litorâneas nordestinas, onde os ventos alcançam grandes velocidades (período do inverno) e há presença de alta irradiância solar (período da primavera). Ainda, por se complementar uma com a outra, haja visto que os ventos são mais intensos durante a noite e, logicamente, a irradiância solar ocorre durante o dia. ${ }^{12,13}$

O problema é que a injeção dessas energias produzidas pelas "micros" usinas geradoras podem ocasionar incertezas probabilísticas ao sistema de distribuição e transmissão das concessionárias. ${ }^{5,7,8}$

Quando ocorre a injeção de outras fontes de energia no sistema de distribuição, é preciso que se conheçam as incertezas probabilísticas para garantir a confiabilidade no sistema, e uma das formas de calcular essas incertezas é a simulação pelo Método Monte Carlo. ${ }^{10}$

$\mathrm{O}$ método Monte Carlo (MMC) é qualquer método de uma classe de métodos estatísticos que se baseiam em amostragens aleatórias massivas para obter resultados numéricos, isto é, repetindo sucessivas simulações um elevado número de vezes, para se calcular probabilidades heuristicamente, tal como se, de fato, se registrassem os resultados reais em jogos de casino (daí o nome). Este tipo de método é utilizado em simulações estocásticas com diversas aplicações em áreas como a física, a matemática, a biologia e outras ciências. ${ }^{6}$

O Método de Monte Carlo é a designação geral para a simulação estocástica, usando números aleatórios, a partir de qualquer distribuição de probabilidade para avaliar de forma numérica um modelo matemático que permita estimar o comportamento de um sistema ou um processo que envolve variáveis estocásticas. A principal vantagem da Simulação de Monte Carlo é que esta oferece uma solução robusta e eficiente para avaliação dos sistemas. A simulação de Monte-Carlo utilizada é não-sequencial, pois os números aleatórios gerados não formam uma sequência para definir outras variáveis. ${ }^{4}$

Portanto, o objetivo deste trabalho é avaliar a confiabilidade de um sistema fictício de geração, por meio da implementação da Simulação de Monte Carlo. O sistema fictício de geração da companhia energética é composto por: - (a) geração firme: 2 (duas) usinas térmica a gás de 30 MW (G1 e G2) e 1(uma) usina térmica a óleo combustível de $20 \mathrm{MW}$ (G3); - (b) fontes renováveis: 1 (um) parque eólico de $4 \mathrm{MW}$ e 1 (uma) usina fotovoltaica de $7 \mathrm{MW}$.

\section{Material e Métodos}

Os dados utilizados neste artigo são provenientes de um trabalho realizado na disciplina de Tópicos em Sistemas de Energia Elétrica, do programa de pós-graduação (mestrado e doutorado) da Universidade Federal de Goiás - Escola de Engenharia Elétrica, Mecânica e de Computação.

Para tanto, foram considerados sistema de geração fictício de uma companhia elétrica compostos por: 
a. geração firme - 2 (duas) usinas térmicas a gás (G1 e G2) com produção de $30 \mathrm{MW}$, e 1 (uma) usina térmica a óleo combustível (G3), com produção de $20 \mathrm{MW}$. Os modelos probabilísticos de confiabilidade estão apresentados na Tabela 1.

b. geração com fontes renováveis: - 1 (um) parque eólico com capacidade nominal de 4MW e 1 (uma) usina fotovoltaica com capacidade nominal de $7 \mathrm{MW}$. Os dados para simulação das cargas de energias renováveis foram extraídos do site Instituto Nacional de Meteorologia(INMET). Para geração eólica, foram utilizados dados da velocidade do vento do município de Touros - Rio Grande do Norte, e para geração da fotovoltaica foram consideradas as irradiâncias solares de Florianópolis - capital do estado de Santa Catarina, entre os dias 15/09 a 22/09/2017.

Para as simulações, foi utilizado o software MATLAB R2014b9, e foram levadas em consideração as seguintes proposições.

- Redução dos vetores irradiação solar: eliminação de números negativos e nulos, pois esses números correspondem à geração nos horários entre 23 as 9 horas, ou seja, não há efeitos de irradiação solar. Também, foram eliminados valores negativos e nulos referente à velocidade do vento, ou seja, valores insuficientes para geração de energia eólica.

- Ainda, para as variáveis velocidades do vento e as irradiâncias solares foram realizados os testes de Kolmogorov-Smirnov, a 1\% de probabilidade, e os dados foram ajustados pela função WEIBUL. Sendo assim, foram geradas as Funções de Distribuições Acumuladas (FDP) e para cada um dos vetores foram gerados os gráficos das funções: densidade de probabilidade, de probabilidade acumulada e probabilidade acumulada inversa.

$\mathrm{O}$ valor médio da velocidade dos ventos foi inferior a $10 \mathrm{~m} / \mathrm{s}$. No entanto, foi conseguido ajuste os dados a $1 \%$ de probabilidade. As curvas de distribuição de probabilidade foram normalizadas de forma a manter o comportamento da variável aleatório, e considerando uma escala de valores maior que a real para tornar possível a produção de energia e o dimensionamento adequando dos recursos disponíveis.

Tabela 1. Modelos probabilístico de confiabilidade das unidades geradoras firme.

\begin{tabular}{|c|c|c|c|c|c|c|}
\hline & \multicolumn{3}{|c|}{ Tempo para saída } & \multicolumn{3}{|c|}{ Tempo para restauração } \\
\hline & & $\alpha$ & $\beta$ & & $\alpha$ & $\beta$ \\
\hline G1 & $\bar{\Xi}$ & 0.311 & 0.648 & हี & -3.633 & 1.438 \\
\hline G3 & $B$ & 0.243 & 0.610 & $\stackrel{0}{9}$ & -3.877 & 1.189 \\
\hline
\end{tabular}

A potência elétrica gerada em Watts da turbina eólica foi calculada convertendo os valores das velocidades do vento em potência elétrica por meio da equação $1 .{ }^{14}$

$$
P=\frac{1}{2} \eta C_{p} y\left(\pi R^{2}\right) v^{3}
$$

Em que: $\eta$ é a eficiência do gerador; $C p$ representa o coeficiente aerodinâmico de potência do rotor; $y$ é a massa específica do ar; $\mathrm{R}$ é o raio do rotor da turbina em metros; $v$ é a velocidade dos ventos $\mathrm{em} \mathrm{m} / \mathrm{s}^{2}$.

Foram calculados e gerados os gráficos da FDP da potência gerada dos geradores eólicos com os dados reais. A partir dela, foram obtidos a FDP (novamente a $1 \%$ de probabilidade e ajustes pela função Weibull) e normalizada usando os valores de potência máxima alcançada nas melho res condições de vento. Logo, os dados obtidos foram usados na Simulação Monte Carlo, levando em consideração o valor da capacidade nominal da usina eólica para determinar o cenário de geração.

Para cálculo da modelagem probabilística de geradora fotovoltaica, foi considerada a irradiância solar $\left(\mathrm{W} / \mathrm{m}^{2}\right)$, e a eficiência de conversão de $18 \%$ das células fotovoltaicas sobre a potência injetada na rede da unidade geradora, calculada pela Equação $n^{0} 2$. 


$$
P=\frac{18}{100} G A
$$

Em que: $G$ é a irradiância solar; $A$ é a área total das células fotovoltaicas.

\section{ORDEM DE DESPACHO PARA CÁLCULO DOS ÍNDICES DE CONFIABILIDADE}

Foram despachadas, primeiro, as usinas que utilizam recursos renováveis. Foram assumidos que os componentes elétricos dessas usinas são $100 \%$ confiáveis, ou seja, tem $F O R=0$. Logo, foram despachadas as usinas térmicas (G1, G2 e G3). Para tanto, foi levada em conta a disponibilidade das máquinas pertencentes a cada usina. Observação: Para a modelagem da demanda do sistema, foi considerado um único patamar de carga possível, correspondente ao cenário de demanda máxima de 50 MW.

\section{SIMULAÇÃO MÉTODO MONTE CARLO - SMMC}

A SMMC foi do tipo sequencial, com período de análise e critério de parada igual a um ano (8760 hs). Os índices de confiabilidade do sistema que foram obtidos através da simulação correspondem a: LOLE - expectativa de perda de carga, LOLP probabilidade de perda e EENS - expectativa de perda de energia.

\section{Resultados e Discussão}

A Tabela 2 apresenta o resumo descritivo das variáveis em estudo: velocidade do vento (município de Mossoró $\mathrm{RN}$ ) e irradiação solar (Florianópolis - SC), nos intervalos dos dias 07/07/2017 a 15/07/2017.

Tabela 2. Resumo descritivos das velocidades do vento e irradiância solar

\begin{tabular}{|c|c|c|c|c|}
\hline Variáveis & Maior & Menor & Média & $\begin{array}{c}\text { Desvio } \\
\text { padrão }\end{array}$ \\
\hline $\begin{array}{c}\text { Velocidade do } \\
\text { Vento } \mathrm{m} / \mathrm{s}\end{array}$ & 13,7 & 1,9 & 7,55 & 2,65 \\
\hline $\begin{array}{c}\text { Irradiação W/ } \\
\mathrm{m}^{2}\end{array}$ & 857,22 & 1,4056 & 312,8 & 275,95 \\
\hline
\end{tabular}

Pode-se ser observado que a média da velocidade do vento no município de Touros, no período estudado, foi de $7,55 \mathrm{~m} / \mathrm{s}$. Logo, de acordo com Martins ${ }^{8}$, esse valor médio encontrado inviabiliza a instalação de uma unidade geradora baseada em energia eólica na região, já que a maioria dos geradores eólicos atuais é projetada para entregar potência nominal com velocidades do vento a partir de $10 \mathrm{~m} / \mathrm{s}$. No entanto, após realizar os testes de Kolmogorov-Smirnov, a $1 \%$ de probabilidade, os dados se ajustaram na FDP da curva de distribuição Weibull, Figuras 1 a 3.

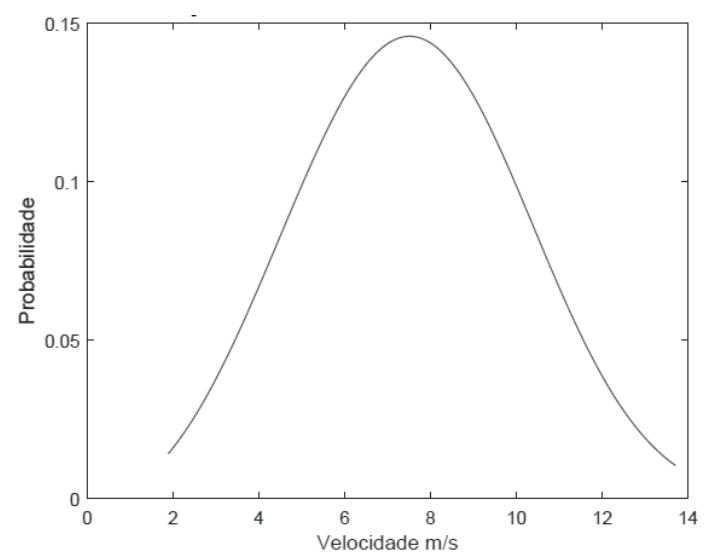

Figura 1. Ajuste da função Weibull - Densidade da Probabilidade para a variável - Vento.

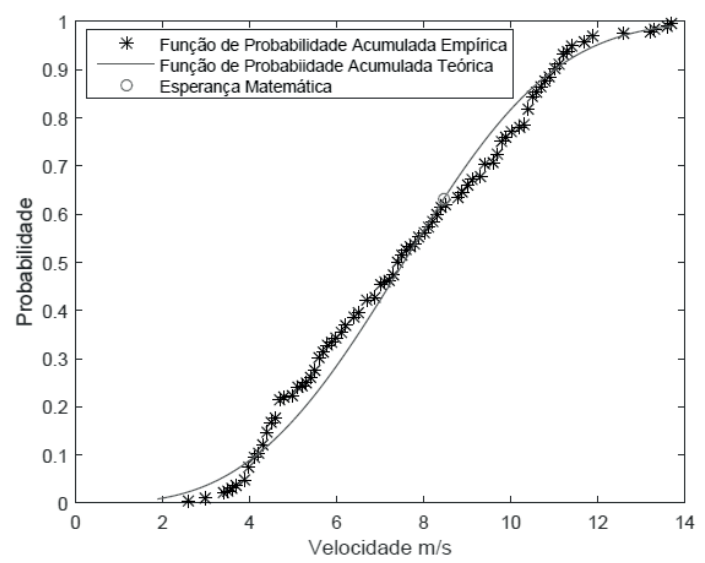

Figura 2. Ajuste da função Weibull - Probabilidade Acumulada para a variável - Vento. 


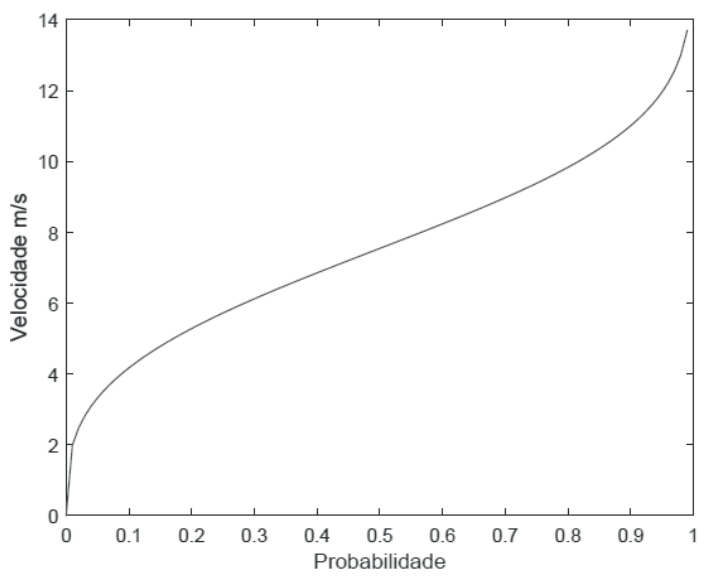

Figura 3. Ajuste da função Weibull - Probabilidade Acumulada Inversa para a variável - Vento.

Ainda, a Tabela 2 apresenta os valores das irradiâncias solares de Florianópolis, sendo $857,22 \mathrm{~W} / \mathrm{m}^{2}$ e $312,8 \mathrm{~W} / \mathrm{m}^{2}$, respectivamente, o maior valor e o valor médio encontrado de acordo com Pinho e Galdino ${ }^{12}$, a potência máxima do módulo nas condições de irradiância é de $800 \mathrm{~W} /$ $\mathrm{m}^{2}$. Dessa forma, os valores médios encontrados estão bem abaixo do esperado para uma boa eficiência de geração energética. Esses baixos valores podem ser explicados em função da data de coleta dos dados (15/09 a 22/09/2017), período que corresponde ao final da estação do inverno, sendo que o período da primavera (setembro a dezembro) é o que apresenta maior irradiância solar ${ }^{13}$. No entanto, este trabalho objetivou complementar as duas formas de geração de energias renováveis (eólica e solar), e para efeito de cálculos, com intuito de fazer complementação entre as duas fontes geradoras, os períodos para coletas de dados deveriam ser os mesmos.

As Figuras 4 a 6 apresentam os resultados dos testes de Kolmogorov-Smirnov, a 1\% de probabilidade, e os dados se ajustados da FDP da curva de distribuição Weibull, para a variável Irradiância.

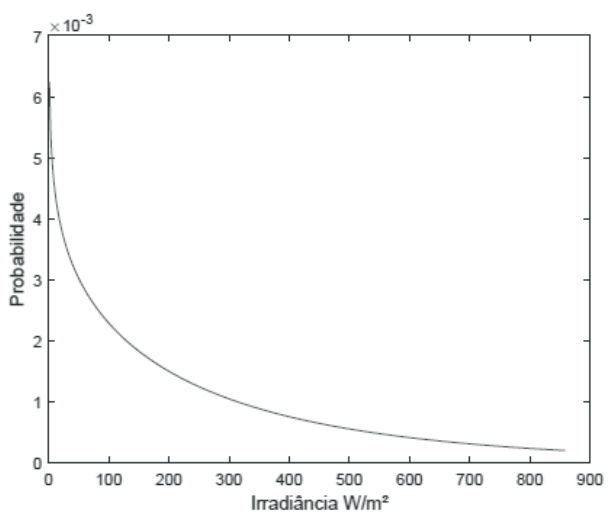

Figura 4. Ajuste da função Weibull - Densidade da Probabilidade para a variável - Irradiância.

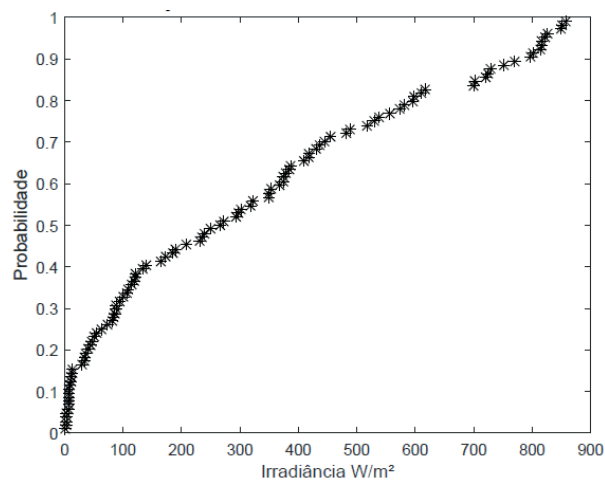

Figura 5. Ajuste da função Weibull - Probabilidade Acumulada para a variável - Irradiância.

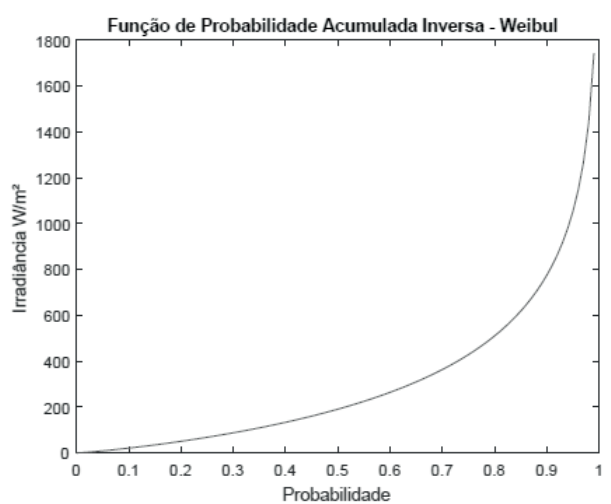

Figura 6. Ajuste da função Weibull - Probabilidade Acumulada Inversa para a variável - Irradiância. 
A potência média calculada e simulada (dados reais), para os geradores eólicos foi $4.6 \mathrm{KW}$ e máxima de $8.3 \mathrm{KW}$. Os cálculos de simulação foram realizados utilizando a Equação 1 (WEG, 2013).

$$
P=\frac{1}{2} \eta C_{p} y\left(\pi R^{2}\right) v^{3}
$$

Em que: $\eta$ é a eficiência do gerador, que considerado igual a 0,$90 ; C p$ representa o coeficiente aerodinâmico de potência do rotor, cujo valor usual assume 0,$45 ; y$ é a massa específica do ar, que considerado $1,225 \mathrm{~kg} / \mathrm{m} 3$; R é o raio do rotor da turbina em metros, que para geradores de até $1 \mathrm{MW}$ é da ordem de $28 \mathrm{~m}$; $v$ é a velocidade dos ventos em $\mathrm{m} / \mathrm{s}^{2}$.

A partir da potência média calculada e simulada para os geradores eólicos (dados reais), foi obtida a FDP acumulada e normalizada, usando o valor da potência máxima alcançada nas melhores condições de vento, conforme apresentada nas Figuras 7 a 9.

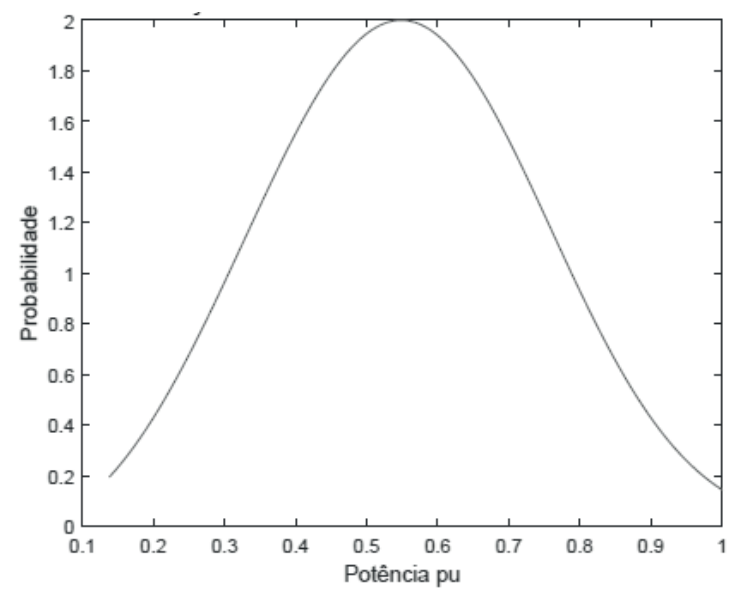

Figura 7. Ajuste da função Weibull - Densidade da Probabilidade para a variável - Potência do Gerador Eólico Normalizado.

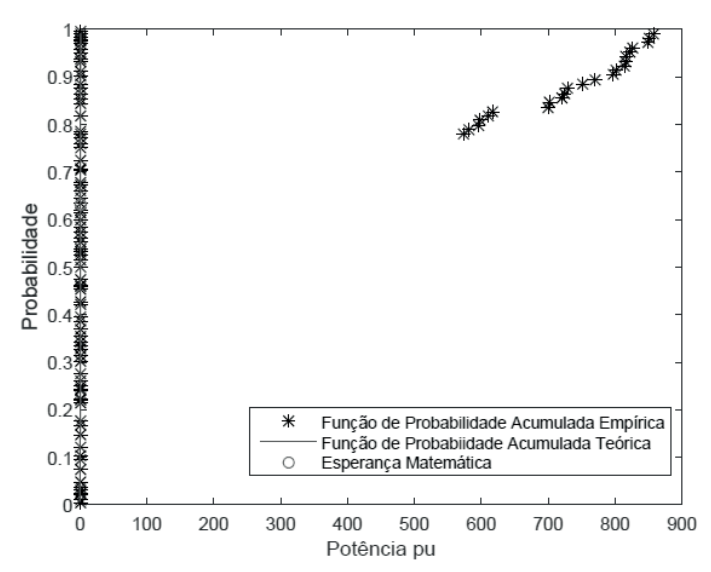

Figura 8. Ajuste da função Weibull - Probabilidade Acumulada para a variável - Potência do Gerador Eólico Normalizado.

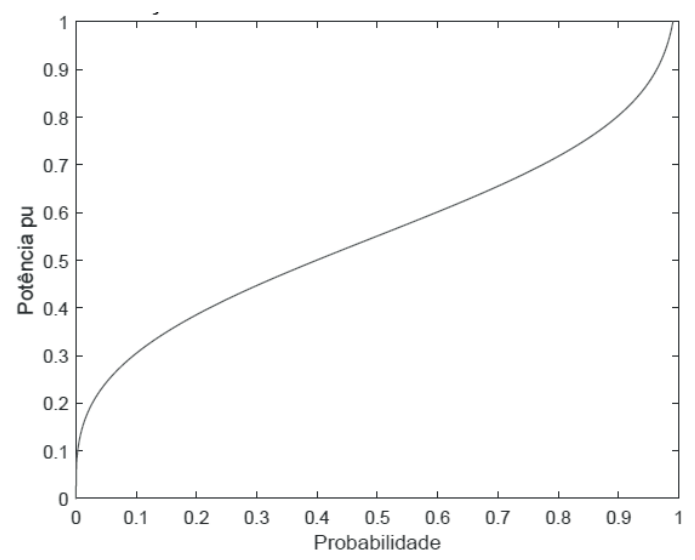

Figura 9. Ajuste da função Weibull - Probabilidade Acumulada Inversa para a variável - Potência do Gerador Eólico Normalizado.

Tirando como referência a potência aleatória em pu, pode ser recalculado a potência em MW do parque eólico. Dessa forma, foi multiplicando-se a potência em pu por $4 \times 106$. Sendo assim, de forma aleatória, foi encontrada a potência em MW do parque eólico.

A partir dos dados fornecidos, foi calculada a área da usina fotovoltaica sendo $61.565,5 \mathrm{~m}^{2}$. E para efeito da modelagem probabilística, calculada de forma randômica, a potência da usina fotovoltaica foi calculada pela Equação $n^{\circ} 2$. 


$$
P=\frac{18}{100} G A
$$

Em que: G é a irradiância solar; A é a área total das células fotovoltaicas, modelada de forma que a usina solar possua potência máxima de $7 \mathrm{MW}$ (potência nominal da usina).

A partir do somatório da potência randômica, gerada pelas fontes renováveis (parque eólico + usina fotovoltaica) e geração firme (de acordo com a ordem de despacho) e da demanda de consumo, pode ser calculado o índice de indisponibilidade pelo método de Simulação Monte Carlo. A SMC foi realizada do tipo sequencial, correspondendo ao período de análise e de parada de 8760 hs ( 1 ano). Os índices de confiabilidade do sistema que foram obtidos de forma randômica, através da simulação: LOLE - expectativa de perda de carga, LOLP - probabilidade de perda e EENS expectativa de perda de energia.

\section{Referências}

3. AGÊNCIA NACIONAL DE ENERGIA ELÉTRICA - ANEEL. Resolução Normativa $N^{\circ}$ 482, de 17 de abril de 2012. Disponível em: <http://www.aneel.gov.br/cedoc/bren2012482.pdf $>$. Acesso em: 18 jun. 2018. 24-28, 2009

4. Assis, A.; Teixeira, O. P. B. Algumas considerações sobre o ensino e a aprendizagem do conceito de energia. Bauru, São Paulo, Ciência e Educação, V.9, nº 1, p 41 a 52, 2003.

5. Atlas de energia elétrica do Brasil / Agência Nacional de Energia Elétrica. 3. ed. - Brasília: Aneel, 2008. 236 p. : il. ISBN: 978-8587491-10-7.

6. Barbosa, F. R. Análise de fluxo de carga com incertezas na geração de demanda - Um estudo de caso. 2013. 76 f. Trabalho de Conclusão de Curso-Curso de Engenharia Elétrica, UFG, Goiânia, 2013.

7. Costa, J. S. Técnicas de Otimizaçao Aplicadas a Sistemas Elétricos de Distribuição. 2008. 107 f. Dissertação (Mestrado) - Curso de Engenharia Elétrica, Ufjf, Juiz de Fora, 2008.

8. Hromkovic, J. Algorithms for hard problems: introduction to combinatorial optimization, randomization, approximation, and heuristics. [S.1.]: Springer-Verlag, London - Berlin - Heidelberg New York, 2001.

9. Marinho, M. H. N. e Aquino, R. R. B. (2009). Oferta de Energia Através da Complementaridade Sazonal Hidro-Eólica. PCH Notícias \& SHP News, n. 40, p.

10. Martins, M. R. P. F.; Trânsito de energia trifásico Probabilístico. 2012. 103 f. Dissertação (Mestrado) - Universidade Técnica de Lisboa, Lisboa, 2012. Disponível em: <https://dspace.ist.utl.pt/ bitstream/2295/1099922/1.pdf>.Acesso em: 10 junho. 2018.
11. Matlab, versão R2012b, Disponível em: www.mathworks.com/ products/matlab.

12. Nunes, R. Apostila - Confiabilidade dos Sistemas Elétricos. Curso de Engenharia de Sistemas Elétricos - Convênio Eletrobrás e Escola Federal de Engenharia de Itajubá. 1980.

13. Olivares, A. Garcia et al.: A global renewable mix with proven technologies and common materials. In: Energy Policy41, (2012),561574, doi:10.1016/j.enpol.2011.11.018.

14. Pinho, J. T.; Galdino, M. A. Manual de Engenharia para Sistemas Fotovoltaicos. Rio de Janeiro, CEPEL - CRESESB, Edição Revisada e Atualizada, Rio de Janeiro 2014.

15. Silva, L. M. Potencial de utilização das energias eólica e solar em Goiânia. 2016. 89 f. Trabalho de Conclusão de Curso - Curso de Engenharia Civil, UFG, Goiânia, 2016.

16. WEG. Geração de Energia Eólica - Tecnologias Atuais e Futuras. Disponível em:<http://ecatalog.weg.net/files/wegnet/WEG-geracaode-energia-eolicatecnologias-atuais e-futuras-artigo-tecnicoportugues-br.pdf $>$. Acesso em: 09 julho. 2018

\section{Márcio J. Dias ${ }^{\star 1,2}$, Murillo V. Santos', Hélio S. Queiroz², Jorge M. A. Costa ${ }^{2}$, Rosemberg F. N. Rodrigues ${ }^{2}$, Lorena F. de Souza ${ }^{3}$, Sonimar R. M. Dias $^{3}, \&$ Vítor S. Menezes ${ }^{3}$}

\author{
${ }^{1}$ Universidade Federal de Goiás - UFG (Campus \\ ESCOLA DE ENGENHARIA ELÉTRICA, \\ MECÂNICA E DE COMPUTAÇÃO - EMC). Av. \\ Universitária, n. ${ }^{\circ} 1488$ - quadra 86 - bloco A - $3^{\circ}$ piso \\ - Setor Leste Universitário, Goiânia - Goiás - CEP: \\ 74605-010. \\ ${ }^{2}$ Centro Universitário de Anápolis - \\ UniEVANGÉLICA, Av. Universitária, Km 3,5, Cidade \\ Universitária, Anápolis-GO, CEP 75083-515. \\ ${ }^{3}$ Faculdade de Tecnologia SENAI Roberto Mange, \\ Rua Prof. Roberto Mange, No 152, CEP 75113-630, \\ Anápolis (GO), CEP 75113-630.
}

*E-mail: marcio.dias@unievangelica.edu.br 
